\title{
OPTIMAL GRADE TRANSITION FOR POLYETHYLENE REACTORS VIA NCO TRACKING
}

\section{INTRODUCTION}

Polyethylene is widely used today in a multitude of products and is produced continuously in gas-phase fluidized-bed reactors. The variety of polyethylene products call for the production of various polymer grades, which can be accomplished by changing the operating conditions of the reactor. Often, a considerable amount of off-specification polymer is produced during grade transitions (Debling et al., 1994). The goal of this work is to analyse and characterize the grade transition problem from the point of view of minimizing the transition time (note that the minimization of the amount of off-spec polymer is very similar).

The grade transition problem has been studied extensively. Debling et al. (1994) tested different grade transition operations using the simulation package POLYRED. McAuley and MacGregor (1992) and Wang et al. (2000) calculated optimal grade transition strategies in a gas-phase fluidized-bed reactor by applying the control

*Correspondence to: Professor D. Bonvin, Laboratoire d'Automatique, École Polytechnique Fédérale de Lausanne, CH-1015 Lausanne, Switzerland. E-mail: dominique.bonvin@epfl.ch vector parameterization (CVP) method to approximate each manipulated variable profile by a series of ramps. Takeda and Ray (1999) also used the CVP method to find optimal grade transitions for a slurry-phase loop reactor. However, in all of the aforementioned works, the cost function is the integral squared error from a pre-defined transition trajectory and not an economic objective. In this work, the optimal grade transition will be considered as a minimal time problem. Another objective is also the interpretation of the various intervals that constitute the optimal solution and relate them to the compromises (in this case none) and the constraints of the optimization problem.

This paper considers the optimization of grade transition via the tracking of the necessary conditions of optimality (NCO tracking). This is accomplished using measurements to directly update the inputs either on-line or on a run-to-run basis. The paper is organized as follows. The next section briefly reviews dynamic optimization and the NCO-tracking scheme. The Process Description section describes the polymerization process and defines the two grades and their corresponding steady-state operating points. The optimal grade transition problem is solved via NCO tracking in the Optimization of Grade Transition section, while conclusions are drawn in the final section. 


\section{OPTIMIZATION VIA NCO TRACKING \\ Dynamic Optimization Problem}

The terminal-cost, finite-time dynamic optimization problem can be formulated mathematically as follows (Bryson and Ho, 1975):

$$
\begin{aligned}
& \min _{u(t), t_{\mathrm{f}}} \phi\left(x\left(t_{\mathrm{f}}\right), t_{\mathrm{f}}\right) \\
& \text { s.t. } \quad \dot{x}=F(x, u), \quad x(0)=x_{0} \\
& S(x, u) \leq 0, \quad T\left(x\left(t_{\mathrm{f}}\right)\right) \leq 0
\end{aligned}
$$

where $\phi$ is a smooth scalar function representing the terminal cost, $x$ the $n$-dimensional vector of states with the known initial conditions $x_{0}, u$ the $m$-dimensional vector of inputs, $S$ the $\zeta$-dimensional vector of path constraints, $T$ the $\tau$-dimensional vector of terminal constraints, $F$ a smooth vector function, and $t_{\mathrm{f}}$ the final time that is finite but can be either fixed or free [the more general case of a free final time is considered in equation (1)].

Using Pontryagin's Minimum Principle, the problem of minimizing the scalar cost functional $\phi$ in equations (1)-(3) can be reformulated as that of minimizing the Hamiltonian function $H(t)$ as follows (Bryson and Ho, 1975):

$$
\begin{aligned}
& \min _{u(t), t_{\mathrm{f}}} H(t)=\lambda^{T} F(x, u)+\mu^{T} S(x, u) \\
& \text { s.t. } \quad \dot{x}=F(x, u), \quad x(0)=x_{0} \\
& \dot{\lambda}^{T}=-\frac{\partial H}{\partial x}, \quad \lambda^{T}\left(t_{\mathrm{f}}\right)=\left.\frac{\partial \bar{\phi}}{\partial x}\right|_{t_{\mathrm{f}}} \\
& \mu^{T} S=0, \quad v^{T} T=0
\end{aligned}
$$

where $\bar{\phi}=\phi+v^{T} T$ is the augmented terminal cost, $\lambda(t) \neq 0$ is the $n$-dimensional vector of adjoint variables, $\mu(\mathrm{t}) \geq 0$ the $\zeta$-dimensional vector of Lagrange multipliers for the path constraints, and $v \geq 0$ the $\tau$-dimensional vector of Lagrange multipliers for the terminal constraints.

Since the solution of a dynamic optimization problem is typically discontinuous and consists of various intervals, the NCO include several parts that correspond to meeting the active constraints and zeroing certain sensitivities, both during the run and at final time (Bryson and Ho, 1975). The constrained part is given by equation (7). The sensitivity part of the NCO is $H_{\mathrm{u}}=\partial H / \partial u=0$, which implies:

$$
\frac{\partial H(t)}{\partial u}=\lambda^{T} \frac{\partial F}{\partial u}+\mu^{T} \frac{\partial S}{\partial u}=0
$$

For a free-terminal-time problem, an additional condition, referred to as the transversality condition, needs to be satisfied:

$$
\frac{\partial \Phi}{\partial t_{\mathrm{f}}}=\left.\frac{\partial \bar{\phi}}{\partial t}\right|_{t_{\mathrm{f}}}+H\left(t_{\mathrm{f}}\right)=0
$$

where $\quad \Phi=\bar{\phi}+\int_{0}^{t_{\mathrm{f}}} H(t) \mathrm{d} t=\phi+v^{T} T+\int_{0}^{t_{\mathrm{f}}}\left[\lambda^{T}(t) F+\right.$ $\left.\mu^{T}(t) S\right] \mathrm{d} t$ is an augmented cost that includes both path and terminal components.
Table 1. Separation of the NCO into four distinct parts.

\begin{tabular}{lcr}
\hline & $\begin{array}{c}\text { Path } \\
\text { objectives }\end{array}$ & $\begin{array}{r}\text { Terminal } \\
\text { objectives }\end{array}$ \\
\hline Constraints & $\mu^{T} S=0$ & $\nu^{T} T=0$ \\
Sensitivities & $H_{\mathrm{u}}=0$ & $\Phi_{\mathrm{t}_{\mathrm{f}}}=0$ \\
\hline
\end{tabular}

The NCO equations (7)-(9) can be rewritten in the partitioned form of Table 1 by separating:

- the conditions linked to the active constraints from those related to sensitivities (first and second rows in Table 1);

- the conditions linked to path objectives from those related to terminal objectives (first and second columns in Table 1).

Some of these conditions can be enforced on-line, while the others need several successive runs to be met.

\section{NCO Tracking Using a Solution Model}

NCO tracking adjusts the manipulated variables by means of a decentralized control system in order to track the NCO in face of uncertainty. This way, optimal operation is implemented via feedback without the need for solving a dynamic optimization problem in real time. The real challenge lies in the fact that four different objectives [equations (7)-(9)] are involved in achieving optimality. These path and terminal objectives are linked to active contraints (7) and to sensitivities (8) and (9). Hence, it becomes important to appropriately parameterize the inputs using time functions and scalars and assign them to the different objectives. This assignment, which corresponds to choosing the solution model, is a way of looking at the NCO through the inputs. The generation of a solution model includes two main steps: (1) input dissection and determination of the fixed and free parts of the inputs based on the effect of uncertainty, and (2) linking the input free parts to the NCO.

\section{Input dissection}

The generation of a solution model typically starts with numerical optimization of a nominal process model. The resulting optimal solution is analyzed for several uncertainty realizations. In some of the intervals, e.g., in intervals where the inputs are at their bounds, the inputs are independent of the prevailing uncertainty and thus can be applied open loop. The corresponding input elements of the solution model are considered as fixed. In other intervals, the inputs are affected by uncertainty and need to be adjusted for optimality. All the input elements affected by uncertainty constitute the decision variables of the optimization problem. These consist of time functions or arcs, $\eta(t)$, and switching times. In addition, certain arcs can be parameterized, thereby turning an infinite-dimensional decision variable into a few scalar decision variables. The parameter vector that includes the switching times and these additional parameters will be denoted generically as $\pi$. Hence, the inputs can be written as $u(t)=\mathcal{U}(\eta(t), \pi)$. 


\section{Linking decision variables to NCO}

The next step consists of providing a link between the decision variables and the NCO. The active state constraints determine certain time functions and the active terminal constraints certain scalar parameters. The remaining decision variables are used to meet all path and terminal sensitivities. Through this assignment, a distinctive label can be tagged on the various input elements as follows:

- The $P C$ variables $\eta_{\mathrm{PC}}(t)$ and $\pi_{\mathrm{PC}}$ vary with uncertainty and can be adjusted by making the corresponding path constraints active.

- The TC variables $\eta_{\mathrm{TC}}(t)$ and $\pi_{\mathrm{TC}}$ vary with uncertainty and can be adjusted by making the corresponding terminal constraints active.

- The PS variables $\eta_{\mathrm{PS}}(t)$ vary with uncertainty and need path sensitivity measurement/estimation for adaptation.

- The $T S$ variables $\pi_{\text {TS }}$ vary with uncertainty and need terminal sensitivity measurement/estimation for adaptation.

An important assumption for this 'labeling scheme' is that the set of active constraints is correctly determined and does not vary with uncertainty. Fortunately, this restrictive assumption can often be relaxed by considering a superstructure for the constraints as will be discussed later. The update of the PC and TC variables are based on path and terminal constraint measurements, respectively. The update equations for the PS and TS variables are based on sensitivity evaluation involving measurements as well. The reader is referred to Srinivasan and Bonvin (2004) for details.

\section{PROCESS DESCRIPTION}

In this study, the polymerization of ethylene in a fluidized-bed reactor with a heterogeneous Ziegler-Natta catalyst is considered (Choi and Ray, 1985; Kiparissides, 1996). A schematic diagram of the reactor system is shown in Figure 1. Ethylene, hydrogen, inert (nitrogen) and catalyst are fed continuously to the reactor. The gas phase consisting of ethylene, hydrogen and nitrogen

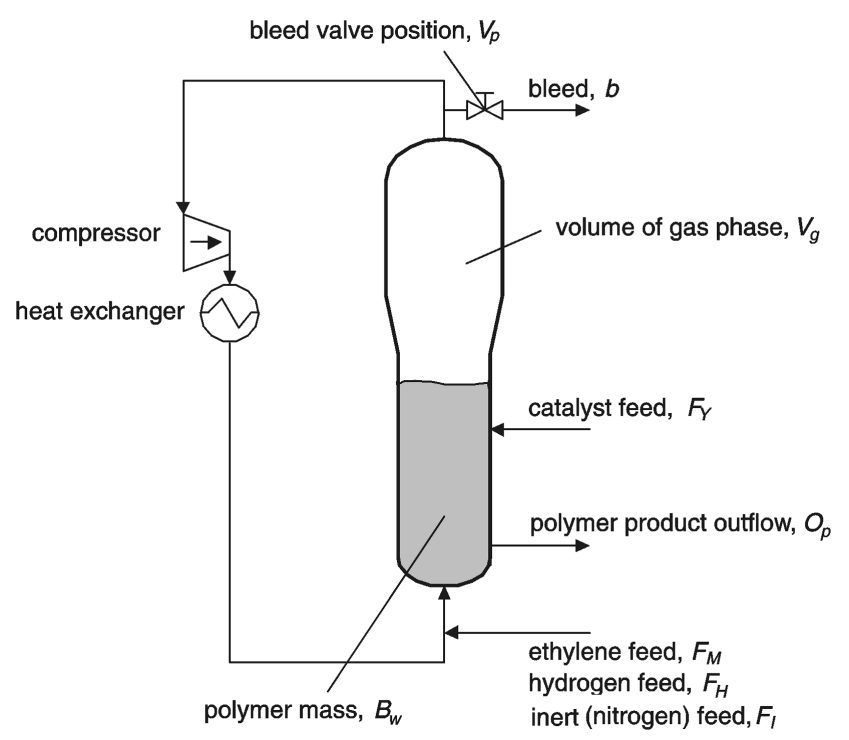

Figure 1. Gas-phase fluidized-bed polyethylene reactor. provides the fluidization of the polymer bed and transports heat out of the reactor through a recycling system. A compressor pumps the recycle gases through a heat exchanger and back to the bottom of the reactor. Fresh feed is added to the recycle stream before entering the reactor. Since the single pass conversion of ethylene in the reactor is usually low $(1-4 \%)$, the recycle stream is much larger than the inflow of fresh feed. Excessive pressure and impurities are removed from the system in a bleed stream at the top of the reactor. Fluidized polymer product is removed from the base of the reactor through a discharge valve. The rate at which the product is removed is adjusted by a bed-level controller that keeps the bed level, i.e., the polymer mass in the reactor, at the desired set point. A simplified first-principles model based on the work of McAuley et al. (1995) and McAuley and MacGregor (1991) is detailed in Gisnas et al., 2003.

During steady-state production of polyethylene, the operating conditions are chosen so as to maximize the outflow rate of polymer of desired grade, while meeting operational and safety requirements. The optimal operating conditions for the two grades $\mathrm{A}$ and $\mathrm{B}$ have been determined in Gisnas et al. (2003) by solving a static optimization problem. These conditions are presented in Table 2 along with the upper and lower bounds used in the optimization. Though, in principle, $V_{\mathrm{p}}$ can be manipulated between 0 and 1 , it is preferred in industry to have a non-zero bleed at steady state to be able to handle impurities. Hence, $V_{\mathrm{p}, \min }=0.5$ is used here. Clearly, increasing $F_{\mathrm{M}}$ increases the production of polyethylene, and thus $F_{\mathrm{M}}$ is maximum. The pressure is at its lower bound in order to minimize the waste of monomer through the bleed, which fixes $F_{\mathrm{I}} . F_{\mathrm{Y}}$ is maximum to increase productivity, $F_{\mathrm{H}}$ is determined from the melt index requirement, $O_{\mathrm{p}}$ is set to keep the polymer mass at its reference value, and the bleed valve position $V_{\mathrm{p}}$ is minimum. Thus, for steady-state optimal operation, the six input variables are determined by six active constraints.

\section{OPTIMIZATION OF GRADE TRANSITION}

\section{Formulation of the Optimization Problem}

The objective is to minimize the transition time $t_{\text {trans }}$ to go from grade $a$ to grade $b$. Among the six inputs, only $F_{\mathrm{H}}$ and $O_{\mathrm{p}}$ are considered as decision variables, while the others are

Table 2. Optimal operating conditions and active constraints for grades A and $\mathrm{B}$, as well as upper and lower bounds used in steady-state optimization $\left(M I_{\mathrm{c}}\right.$ is the cumulative melt index, $B_{\mathrm{w}}$ the polymer mass in the reactor bed, $P$ the pressure of the gas phase, $F_{\mathrm{H}}, F_{\mathrm{I}}, F_{\mathrm{M}}$ and $F_{\mathrm{Y}}$ the fresh feeds of hydrogen, inert (nitrogen), monomer (ethylene) and catalyst, respectively, $V_{\mathrm{p}}$ the bleed valve position, and $O_{\mathrm{p}}$ the outflow rate of polymer product from the reactor).

\begin{tabular}{lccccc}
\hline & $\begin{array}{l}\text { Lower } \\
\text { bound }\end{array}$ & $\mathrm{A}$ & $\mathrm{B}$ & $\begin{array}{r}\text { Upper } \\
\text { bound }\end{array}$ & $\begin{array}{c}\text { Active } \\
\text { constraint }\end{array}$ \\
\hline$M I_{\mathrm{c}, \mathrm{ref}}(\mathrm{g} / 10 \mathrm{~min})$ & & 0.009 & 0.09 & & \\
$B_{\mathrm{w}, \mathrm{ref}}\left(10^{3} \mathrm{~kg}\right)$ & 20 & 20 & 70 & & \\
$P(\mathrm{~atm})$ & 0 & 1.1 & 15 & 70 & $M I_{\mathrm{c}, \mathrm{ref}}$ \\
$F_{\mathrm{H}}\left(\mathrm{kg} \mathrm{h}^{-1}\right)$ & $\mathbf{0}$ & 495 & 281 & 500 & $F_{\mathrm{I}, \min }$ \\
$F_{\mathrm{I}}\left(\mathrm{kg} \mathrm{h}^{-1}\right)$ & 0 & 30 & 30 & $\mathbf{3 0}$ & $F_{\mathrm{M}, \max }$ \\
$F_{\mathrm{M}}\left(10^{3} \mathrm{~kg} \mathrm{~h}^{-1}\right)$ & 0 & 10 & 10 & $\mathbf{1 0}$ & $F_{\mathrm{Y}, \max }$ \\
$F_{\mathrm{Y}}\left(10^{-3} \mathrm{kmol} \mathrm{h}^{-1}\right)$ & $\mathbf{0 . 5}$ & 0.5 & 0.5 & 1 & $V_{\mathrm{p}, \min }$ \\
$V_{\mathrm{p}}\left(10^{3} \mathrm{~kg} \mathrm{~h}^{-1}\right)$ & 21 & 29.86 & 29.84 & 39 & $B_{\mathrm{w}, \mathrm{ref}}$ \\
$O_{\mathrm{p}}$ & & & & &
\end{tabular}


kept at active bounds (see quantities in bold in Table 2): The bounds for $F_{\mathrm{M}}, F_{\mathrm{Y}}$ and $V_{\mathrm{p}}$ correspond to values for optimal steady-state operation and that for $F_{\mathrm{I}}$ serves to keep the pressure as low as possible during transition. However, in contrast to steady-state operation, the polymer mass $B_{\mathrm{w}}$ is allowed to vary between 50 and $84\left(10^{3} \mathrm{~kg}\right)$.

The dynamic optimization problem can be stated mathematically as follows:

$$
\min _{F_{\mathrm{H}}(t), O_{\mathrm{p}}(t), t_{\text {trans }}} J=t_{\text {trans }}
$$

s.t. dynamic system

$$
\begin{aligned}
& F_{\mathrm{H}, \text { min }} \leq F_{\mathrm{H}}(t) \leq F_{\mathrm{H}, \max } \\
& O_{\mathrm{p}, \text { min }} \leq O_{\mathrm{p}}(t) \leq O_{\mathrm{p}, \max } \\
& B_{\mathrm{w}, \min } \leq B_{\mathrm{w}}(t) \leq B_{\mathrm{w}, \max } \\
& M I_{\mathrm{c}}\left(t_{\text {trans }}\right)=M I_{\mathrm{c}, \text { ref }} \\
& M I_{\mathrm{i}}\left(t_{\text {trans }}\right)=M I_{\mathrm{c}, \text { ref }} \\
& B_{\mathrm{w}}\left(t_{\text {trans }}\right)=B_{\mathrm{w}, \text { ref }}
\end{aligned}
$$

where $M I_{\mathrm{i}}$ is the instantaneous melt index:

$$
M I_{\mathrm{i}}=k_{\mathrm{T}}\left(k_{1}+k_{2} \frac{\left[H_{2}\right]}{[M]}\right)^{3.5}
$$

with $k_{\mathrm{T}}$ the chain transfer rate constant, $k_{1}$ and $k_{2}$ melt index constants, and $\left[\mathrm{H}_{2}\right]$ and $[M]$ the concentrations of hydrogen and monomer (ethylene), respectively.

\section{Optimal Nominal Solution}

Optimization problem (10) is solved numerically using the optimization toolbox of MATLAB. The optimal solution presented in Figure 2 can be interpreted as follows:

- $F_{\mathrm{H}}$ is maximum initially in order to increase $M I_{\mathrm{i}}$ as quickly as possible through an increase of $\left[H_{2}\right]$ [equation (11)]. $F_{\mathrm{H}}$ is later switched to its lower bound so as to meet the terminal constraint $M I_{\mathrm{i}}$ $\left(t_{\text {trans }}\right)=M I_{\text {c,ref }}$.

- $O_{\mathrm{p}}$ is minimum initially to help increase $M I_{\mathrm{i}}$, which can be accomplished through a decrease of $[M]$ [equation (11)]. For this, more catalyst is needed, i.e., $Y$ is increased. This is achieved by removing less catalyst
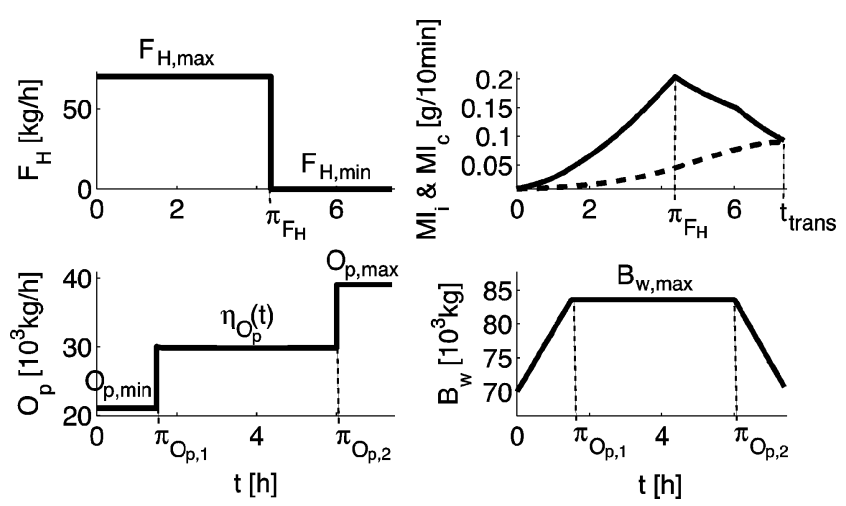

Figure 2. Optimal profiles for the transition $A \rightarrow B\left(M I_{\mathrm{i}}\right.$ continuous, $M I_{\mathrm{c}}$ dashed). with the product, i.e., by closing the outlet valve, $O_{\mathrm{p}}=$ $O_{\mathrm{p}, \min }$. However, closing the outlet valve increases the polymer mass. When $B_{\mathrm{w}}$ reaches its upper bound, $O_{\mathrm{p}}$ is adjusted to keep this constraint active, which gives the second arc $\eta_{\mathrm{O}_{\mathrm{p}}}(t)$. Finally, $O_{\mathrm{p}}$ is maximum in order to decrease the polymer mass and meet the terminal constraint $B_{\mathrm{w}}\left(t_{\text {trans }}\right)=B_{\mathrm{w}, \text { ref }}$.

\section{Solution Model}

- Input dissection

(1) Structure detection: The nominal solution presented in Figure 2 consists of input arcs determined by either input bounds $\left(F_{\mathrm{H}, \max }, F_{\mathrm{H}, \min }, O_{\mathrm{p} \text {,min }}\right.$ and $\left.O_{\mathrm{p}, \max }\right)$ or state constraint $\left(B_{\mathrm{w}, \max }\right)$, but it does not contain sensitivity-seeking arcs.

(2) Determination of fixed and free input parts: The fixed parts consists of the inputs at their bounds $\left(F_{\mathrm{H}, \text { max }}, F_{\mathrm{H} \text {,min }}, O_{\mathrm{p}, \text { min }}\right.$ and $\left.O_{\mathrm{p}, \text { max }}\right)$, while the free parts are the state-constrained arc $\eta_{\mathrm{O}_{\mathrm{p}}}(t)$ and the switching times $\pi_{\mathrm{F}_{\mathrm{H}}}, \pi_{\mathrm{O}_{\mathrm{p}, 1}}$, and $\pi_{\mathrm{O}_{\mathrm{p}, 2}}$.

(3) Parameterization of the decision variables: Since there is no sensitivity-seeking arc to be parameterized, the parameter vector $\pi$ contain only the switching times $\pi_{\mathrm{F}_{\mathrm{H}}}, \pi_{\mathrm{O}_{\mathrm{p}, 1}}, \pi_{\mathrm{O}_{\mathrm{p}, 2}}$ and the transition time $t_{\text {trans }}$.

- Links to NCO

(1) Links to state constraints: The arc $\eta_{\mathrm{O}_{\mathrm{p}}}(t)$ is linked to the state constraint $B_{\mathrm{w}}(t)=B_{\mathrm{w}, \max }$. The parameter $\pi_{\mathrm{O}_{\mathrm{p}, 1}}$ is determined implicitly by $B_{\mathrm{w}}(t)$ reaching its upper bound.

(2) Links to terminal constraints: The remaining parameters $\pi_{\mathrm{F}_{\mathrm{H}}}, \pi_{\mathrm{O}_{\mathrm{p}, 2}}$ and $t_{\text {trans }}$ are linked to the terminal constraints $M I_{\mathrm{i}}\left(t_{\text {trans }}\right)=M I_{\mathrm{c}, \text { ref }}, \mathrm{B}_{\mathrm{w}}\left(t_{\text {trans }}\right)=B_{\mathrm{w}, \text { ref }}$ and $M I_{\mathrm{c}}\left(t_{\text {trans }}\right)=M I_{\mathrm{c}, \text { ref }}$, respectively.

(3) Links to path and terminal sensitivities: There are neither path nor terminal sensitivities in this problem since all parameters and arcs have already been assigned to state and terminal constraints.

Having dissected the inputs and linked the free parts to the NCO, the solution model can be written formally as follows:

$$
\begin{aligned}
& F_{\mathrm{H}}(t)=\left\{\begin{array}{l}
F_{\mathrm{H}, \max } \quad \text { for } 0 \leq t<\pi_{\mathrm{F}_{\mathrm{H}}} \\
F_{\mathrm{H}, \min } \text { for } \pi_{\mathrm{F}_{\mathrm{H}}} \leq t \leq t_{\text {trans }}
\end{array}\right. \\
& O_{\mathrm{p}}(t)=\left\{\begin{array}{l}
O_{\mathrm{p}, \min } \text { for } 0 \leq t<\pi_{\mathrm{O}_{\mathrm{p}, 1}} \\
\mathcal{K}_{\eta \mathrm{O}_{\mathrm{p}}}\left(B_{\mathrm{w}, \max }-B_{\mathrm{w}}(t)\right) \text { for } \pi_{\mathrm{O}_{\mathrm{p}, 1}} \leq t<\pi_{\mathrm{O}_{\mathrm{p}, 2}} \\
O_{\mathrm{p}, \max } \text { for } \pi_{\mathrm{O}_{\mathrm{p}, 2} \leq t \leq t_{\text {trans }}}
\end{array}\right. \\
& \pi_{\mathrm{O}_{\mathrm{p}, 1}}=t \quad \text { with } \quad B_{\mathrm{w}}(t)=B_{\mathrm{w}, \max } \text { and } \\
& \pi_{\mathrm{F}_{\mathrm{H}}}= \mathcal{R}_{\pi_{\mathrm{F}}}\left(M I_{\mathrm{c}, \text { ref }}-M I_{\mathrm{i}}\left(t_{\text {trans }}\right)\right) \\
& \pi_{\mathrm{O}_{\mathrm{p}, 2}}= \mathcal{R}_{\pi_{\mathrm{O}, 2}}\left(B_{\mathrm{w}, \text { ref }}-B_{\mathrm{w}}\left(t_{\text {trans }}\right)\right) \\
& t_{\text {trans }}= \mathcal{R}_{t_{\text {trans }}}\left(M I_{\mathrm{c}, \text { ref }}-M I_{\mathrm{c}}\left(t_{\text {trans }}\right)\right)
\end{aligned}
$$

where $\mathcal{K}$ and $\mathcal{R}$ are on-line and run-to-run controllers, respectively, and $t_{-}$represents a time just prior to $t$. 


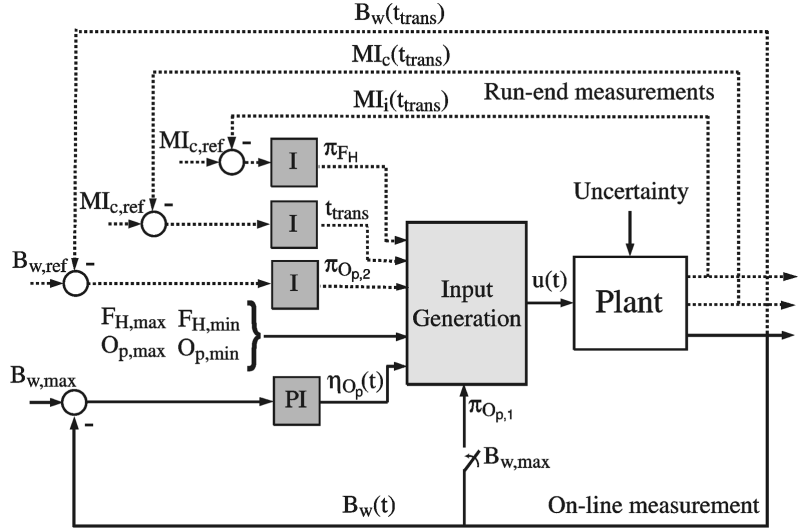

Figure 3. Control scheme for the adaptation of the solution model.

\section{Adaptation of the Solution Model}

The adaptation of the free variables can be implemented with a decentralized control scheme, as illustrated in Figure 3. The adaptation of the constraint-seeking $\eta_{\mathrm{O}_{\mathrm{p}}}(t)$ is implemented on-line using a PI controller, while the adaptation of the switching times $\pi_{\mathrm{F}_{\mathrm{H}}}, \pi_{\mathrm{O}_{\mathrm{p}, 2}}$ and $t_{\text {trans }}$ is performed on a run-to-run basis using discrete integral controllers.

\section{Simulated NCO-Tracking Results}

It is assumed that uncertainty is present in the plant in the form of time-varying kinetic coefficients, which may correspond to a variation of catalyst efficiency with time. This information on parametric variations is, of course, not revealed to the measurement-based optimization algorithms. If this information were available, the minimal transition time would be $J^{*}=7.36 \mathrm{~h}$.

The NCO-tracking results of Table 3 call for several remarks:

(1) Direct application of the nominal optimal inputs is infeasible as it violates the path constraint $B_{\mathrm{w}}(t) \leq$ $B_{\mathrm{w}, \max }$ as well as terminal constraints.

(2) The run-to-run nature of the control scheme given in Figure 3 results in the first two runs to be infeasible with respect to the terminal constraints. However, this could be corrected using PID controllers to drive the process from the state at $t_{\text {trans }}$ to the feasible region, assuming $M I_{\mathrm{i}}$ and $M I_{\mathrm{c}}$ are measured on-line. Thus, in the case of infeasible solutions due to violation of terminal constraints, the cost $J$ also includes the time needed to become feasible.

(3) Convergence is achieved within three runs. Furthermore, there is already considerable cost improvement after two runs.
(4) The run-to-run scheme is here of multivariable nature: the three decision variables $\bar{\pi}=\left[\pi_{\mathrm{F}_{\mathrm{H}}}, \pi_{\mathrm{O}_{\mathrm{p}, 2}}, t_{\text {trans }}\right]^{T}$ are used to meet the three terminal constraints $M I_{\mathrm{i}}\left(t_{\text {trans }}\right)=$ $M I_{\text {c,ref }}, \mathrm{B}_{\mathrm{w}}\left(t_{\text {trans }}\right)=B_{\mathrm{w}, \text { ref }}$ and $M I_{\mathrm{c}}\left(t_{\text {trans }}\right)=M I_{\mathrm{c}, \text { ref }}$. The pairing was done intuitively as indicated in the solution model (12). A decoupling scheme based on the sensitivity matrix $\partial T j / \partial \bar{\pi}$ could be implemented to speed up convergence (not needed here in this noisefree case).

(5) In industrial practice, though grade changes are made frequently, the same grade change is not necessarily repeated. This makes the run-to-run aspect less appealing. Thus, it is of high interest to meet the most important (from a cost point of view) terminal constraints on-line as proposed in Srinivasan and Bonvin (2004).

(6) The advantage of this optimization approach is that only measurement of bed level is required on-line.

\section{CONCLUSIONS}

Using a tendency model for a gas-phase fluidized-bed reactor, it was possible to show that the optimal grade transition problem is completely determined by path and terminal constraints. The resulting solution model links the free (adjustable) elements of the manipulated variables to appropriate measurements. This way, it has been possible to adjust the inputs so that they are nearly optimal for a simulated uncertain reality. This calls for important observations with regard to the applicability of NCO tracking to experimental processes:

- Optimality is seen in the context of approximation. The objective is to approach optimality for a complex and poorly-modelled reality. The true optimal solution is unknown. At best, that obtained from some approximate model could be determined. The approach taken here is to assume that the shape of the optimal inputs (i.e., the main arcs related to active constraints and compromises in the optimization problem) obtained from the simplified nominal model is sufficient to approximate the true optimal inputs. Then, process measurements are used to adjust the inputs towards optimality.

- The approach relies on the measurement or estimation of constrained quantities and sensitivities. Hence, if these quantities are not directly available through measurements, it is necessary to estimate them. Note, however, that the state estimation problem does not necessarily need to reconstruct the full state vector, but only those quantities that are involved in the NCO. Furthermore, it is often possible to formulate alternative solution models that are considerably easier to implement (Srinivasan and Bonvin, 2004).

Table 3. Values of the constrained quantities, transition time and cost $J$ as functions of the numbers of runs; no measurement noise is considered (the quantities in bold indicate a constraint violation; the costs need to be compared to the ideal value $J^{*}=7.36 \mathrm{~h}$ ).

\begin{tabular}{lcccrr}
\hline $\begin{array}{l}\text { Number of } \\
\text { runs }\end{array}$ & $\begin{array}{c}M I_{\mathrm{c}}\left(t_{\text {trans }}\right)(\mathrm{g} 10 \mathrm{~min}) \\
M I_{\mathrm{c}, \text { ref }}=0.090\end{array}$ & $\begin{array}{c}M I_{\mathrm{i}}\left(t_{\text {trans }}\right)(\mathrm{g} 10 \mathrm{~min}) \\
M I_{\mathrm{c}, \text { ref }}=0.090\end{array}$ & $\begin{array}{c}B_{\mathrm{w}}\left(t_{\text {trans }}\right)\left(10^{3} \mathrm{~kg}\right) \\
B_{\mathrm{w}, \mathrm{ref}}=70.0\end{array}$ & $\begin{array}{c}t_{\text {trans }} \\
(\mathrm{h})\end{array}$ & $\begin{array}{c}J \\
(\mathrm{~h})\end{array}$ \\
\hline 1 & $\mathbf{0 . 0 9 7}$ & $\mathbf{0 . 0 9 8}$ & $\mathbf{6 9 . 9}$ & 7.45 & 10.39 \\
2 & $\mathbf{0 . 0 9 3}$ & $\mathbf{0 . 0 9 4}$ & $\mathbf{7 0 . 6}$ & 7.39 & 8.88 \\
3 & 0.090 & 0.090 & 70.0 & 7.36 & 7.36 \\
10 & 0.090 & 0.090 & 70.0 & 7.36 & 7.36 \\
\hline
\end{tabular}


- The investigation was performed here in simulation, whereby the solution model obtained from a nominal model was used to reach optimality for a different (socalled perturbed) model. In practice, the perturbed model is the real process itself, which means that a simplified nominal model that is able to characterize the shape of the optimal solution will lead to near optimality for the real process. This seems to be of considerable industrial interest. We have started to implement the NCO-tracking scheme on several experimental set-ups and also on larger industrial processes. Acceptance has exceeded our expectations. The main remarks deal with (1) its ability to improve process operation, while keeping it really simple, and (2) the fact that it helps formalize the qualitative knowledge that the operators or engineers have but cannot implement in a systematic way.

\section{REFERENCES}

Bryson, A.E. and Ho, Y.C., 1975, Applied Optimal Control (Hemisphere, Washington DC, USA).

Choi, K.Y. and Ray, W.H., 1985, The dynamic behaviour of fluidized bed reactors for solid catalysed gas-phase olefin polymerization, Chem Eng Sci, 40: 2261-2279.
Debling, J.A. et al., 1994, Dynamic modeling of product grade transitions for olefin polymerization processes, AIChE J, 40(3): 506-520.

Gisnas, A. et al., 2003, Optimal grade transition for polyethylene reactors, in Process Systems Engineering, 463-468 (Kunming, China).

Kiparissides, C., 1996, Polymerization reactor modeling: a review of recent developments and future directions, Chem Eng Sci, 51(10): $1637-1659$.

McAuley, K.B. et al., 1995, Effects of operating conditions on stability of gas-phase polyethylene reactors, AIChE J, 41(4): 868-879.

McAuley, K.B. and MacGregor, J.F., 1991, On-line inference of polymer properties in an industrial polyethylene reactor, AIChE J, 37(6): $825-835$.

McAuley, K.B. and MacGregor, J.F., 1992, Optimal grade transitions in a gas-phase polyethylene reactor, AIChE J, 38(10): 1564-1576.

Srinivasan, B. and Bonvin, D., 2004, Dynamic optimization under uncertainty via NCO tracking: a solution model approach, in BatchPro Symposium, 17-35 (Poros, Greece).

Takeda, M. and Ray, W.H., 1999, Optimal grade transition strategies for multistage polyolefin reactors, AIChE J, 45(8): $1776-1793$.

Wang, Y. et al., 2000, Optimal grade transition control for polymerization reactors, Comp Chem Eng, 24: 1555-1561.

This paper was presented at the 7th World Congress of Chemical Engineering held in Glasgow, UK, 10-14 July 2005. The manuscript was received 15 December 2004 and accepted for publication after revision 4 April 2005. 
TO: CORRESPONDING AUTHOR

\section{AUTHOR QUERIES - TO BE ANSWERED BY THE AUTHOR}

The following queries have arisen during the typesetting of your manuscript. Please answer these queries by marking the required corrections at the appropriate point in the text.

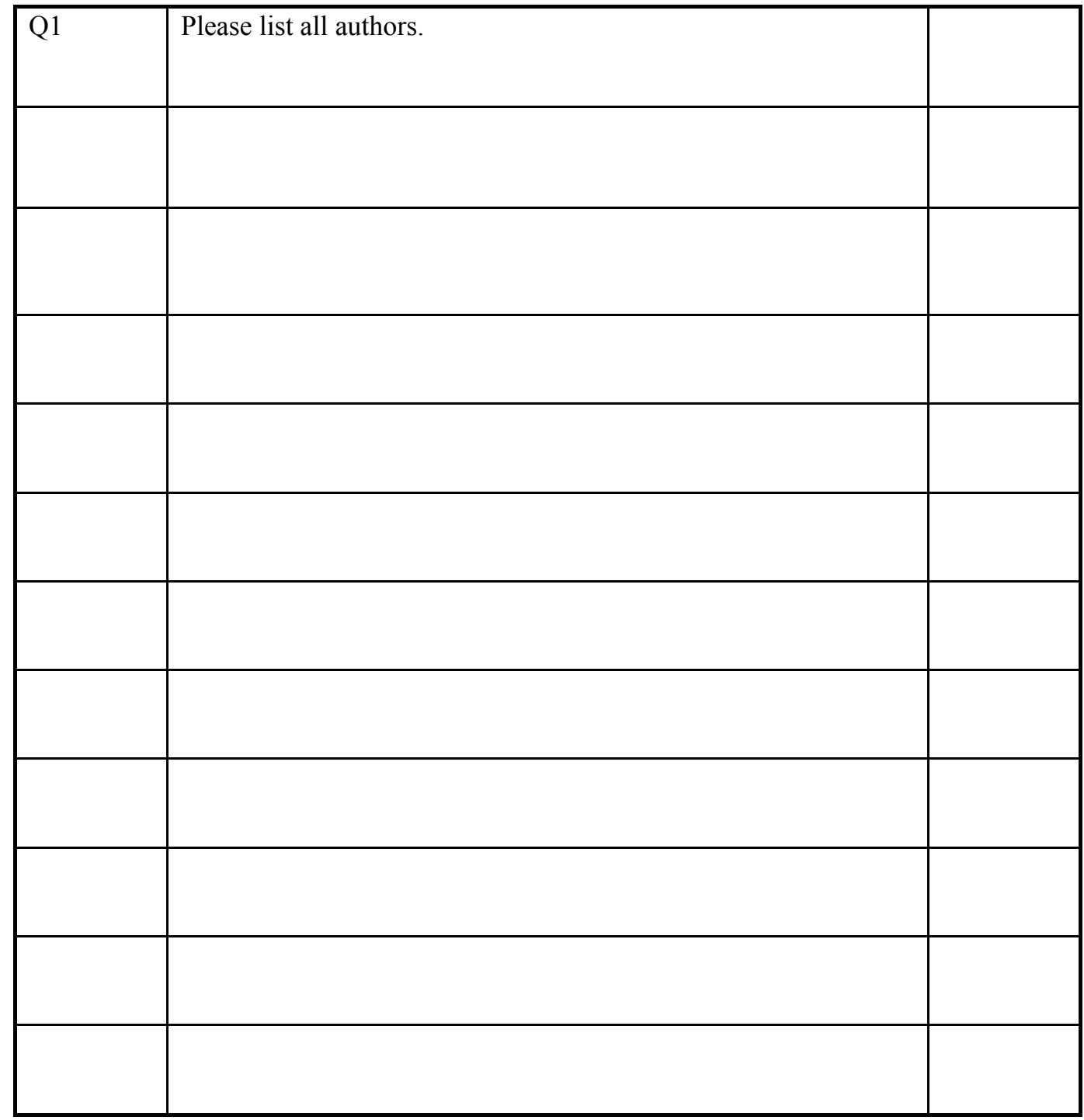

